\title{
Le récit de voyage de Pedro Montengón (1745-1824)
}

\section{Pedro Montengon's Travel History (1745-1824)}

\section{Dame DIOP' 1 (1)}

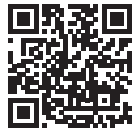

${ }^{1}$ Assistant, Assane Seck University of Ziguinchor, Faculty of Arts and Humanities, Department of LEA (Applied Foreign Languages), Ziguinchor, Sénégal

ORCID: D.D. 0000-0002-7257-5706

Corresponding author:

Dame DIOP,

Assane Seck University of Ziguinchor, Faculty of Arts and Humanities, Department of LEA (Applied Foreign Languages), Ziguinchor, Sénégal E-mail: dame.diop@univ-zig.sn

Submitted: 12.12 .2019

Revision Requested: 14.01.2020 Last Revision Received: 01.04 .2020 Accepted: 08.04.2020

Citation: Diop, D. (2020). Le récit de voyage de Pedro Montengón (1745-1824). Litera, 30(1), 105-119. https://doi.org/10.26650/LITERA2019-0076

\section{RÉSUMÉ}

Cet article montre la place du poète, romancier philosophe et dramaturge, Pedro Montengón (1745-1824), dans la littérature de voyage à travers son best-seller Eusebio (1786), un roman didactique qui réactualise les idées classiques du naufrage en tant que symbole de la destinée de l'homme à l'image de la poésie de Quevedo (1580-1645) grâce à la navigation. Cette dernière reflète la vie de l'homme dans le monde dont l'unique salut réside dans la modestie, la modération et l'humilité, au vu de la vie des sauveteurs pacifique, tranquille et paradisiaque de la grange d'Henrique Myden sur le littoral dans le roman de Montengón. Victimes de l'ambition et de la cupidité, les parents du jeune Eusebio prennent ainsi le risque de traverser la mer dangereuse pour se rendre en Amérique afin d'augmenter leur fortune, avant de faire naufrage au large des côtes de Maryland. Sauvé et dépourvu de toute la richesse familiale à bord du bateau, l'Émile espagnol est destiné à l'apprentissage de la vertu naturelle qui est à la base de la foi chrétienne incarnée par un homme neuf (Eusebio). Formé dans les métiers manuels et pétri de vertu, ce dernier sort indemne de la mer lors de ses voyages maritimes.

Mots-clés: Récit de voyage, mer, littoral, naufrage, destinée

\section{ABSTRACT}

This article demonstrates the place of the poet, novelist-cum-philosopher and playwright, Pedro Montengón (1745-1824), in travel literature through his bestseller Eusebio (1786), a didactic novel that updates the classical ideas of shipwreck as a symbol of human destiny in the image of Quevedo's poetry (1580-1645) via navigation. The latter shows the life of man in the world whose only salvation lies in modesty, moderation and humility, and this is reflected in the lives of the peaceful, quiet and heavenly rescuers of Henrique Myden's coastal barn in Montengón's novel. Victims of ambition and greed, the parents of the young Eusebio took the risk of crossing the dangerous sea to go to America to increase their fortune before being shipwrecked off the coast of Maryland. Saved and deprived of all the family wealth on board the boat, the Spanish Emile is destined to learn the natural virtue that is at the base of the Christian faith embodied in a new man (Eusebio). Trained in manual trades and steeped in virtue, the latter emerged unharmed from the sea during his sea voyages.

Keywords: Travel story, sea, coast, shipwreck, destiny 


\section{EXTENDED ABSTRACT}

This article shows the representation of the sea in the travel narrative of Pedro Montengón (1745-1824) through his didactic novel Eusebio (1786), thanks to the cybernetic space that contains data cues. The main character of this novel is a young man (Eusebio), a survivor of a shipwreck off the coast of Maryland, Pennsylvania, where he begins his education in an adoptive family. He loses his parents, his sister and all his belongings in the shipwreck on his way to America, the New World, where his parents intended to settle in order to improve their wealth. His ambition is held back by the furious wind, the violent storm, the demonic waves, the shipwreck and the death of all the passengers except the young noble Eusebio, who is rescued by another survivor Gil Altano hanging from a mast. We have a description of the hostile and dangerous sea in contrast to the coastline where they saw the servants of a Quaker, a rich businessman (Henrique Myden).

Having examined the significance of the sea, we then examine the details of the staging between the tribulations of the shipwrecked and the gaze of the spectators who end up rescuing them. This process of staging between the shipwrecked and the spectator will allow us to establish a certain correspondence in the representation of the sea and the coastline between Quevedo (1580-1645) and Pedro Montengón (17451824). Indeed, we must point out that while reading Carmen Peraita's article (Peraita, 2002), we realize that both authors have more or less the same ideas that explain the loss of the man, who is a victim of vices such as ambition, easy gain and lack of restraint and which drive him to set sail, as opposed to the virtues (moderation, modesty and humility) of his rescuer (the spectator), one who has a quiet and peaceful life on the coast.

Moreover, it is a philosophy that is conveyed by a literary means (the shipwreck) through a narrative technique, comparing the description of the sea and the coastline. Thus, the idea is to compare these two authors who both treated the issues of their time in the same way, even if they are not from the same period. We will see that for these two writers, the causes of human perdition are the same (ambition, excessiveness and greed) and lead to navigation (Peraita, 2002, p. 185), which is often fatal (Peraita, 2002 , p. 185). The philosophical meaning of the process by which the spectator perceives the shipwreck from a land perspective will be examined (Peraita, 2002, p. 187). In a more existential dimension, the starting point is that one is already on board and there 
is no possibility of abstaining: the outcome for man is only rescue or drowning, according to Blumenberg (quoted by Peraita, 2002, p. 187). In short, boats are the symbol of destiny.

On the other hand, the narrative function of space (functional topography) will allow us to better grasp the meaning of these different spaces in Pedro Montengón's novel, be it the maritime space, the coastline, the coasts, the barn of his adoptive parents facing the sea. This method of analysis based on the mimetical representation of space and on the symbolic or ideological function or dimension of space in correlation with the actions of the characters brings us back to the semiology of space as a meaningful tool that establishes similarities between Montegon and Quevedo from the narrative point of view and that put up front the ideological dimension of space. In fact, the writer from Alicante uses the journey to the New World to highlight, at the same time, his romantic ideas, through the personification of the sea, the furious wind, the clouds, the night, the storm, the shipwreck, and death, updating the classical ideas as a preromantic writer. The boat serves the narrative because the shipwreck provokes "ruptures" of destiny. It is also a meeting place. Again, Montengón's novel uses it both in an ancient way (in reference to the Byzantine novel, that is to say, the succession of events through navigation, the shipwreck allowing the opening of perspectives and encounters) and in a modern way (the boat favours introspection and moral testing).

Finally, our reflection will be articulated around two main axes. Firstly, we will look at the place of Pedro Montengón in travel literature (1.). Then, we will successively study: Quevedo's influence and Montengón's innovative role in the travel narrative (1.1.), the portrayal of the shipwrecked and the rescuers (1.2.) and his pre-romantic ideas through the description of the sea and the coastline (1.3.). Secondly, we will examine the opposition between the coast and the sea (1.4.) and then take a look at the role of ships as a symbol of destiny (2.) by relying on the narrative role of the shipwreck (2.1.), through the journey (2.2.) and the interpolated novels (2.3.). 


\section{Introduction}

Au XVIII siècle se produit une profonde mutation dans la sensibilité. L'Occident découvre peu à peu les beautés de la nature sauvage qui auparavant faisait peur : les descriptions s'attachent à la montagne, à la forêt, à l'océan, auxquels s'attache maintenant une forte charge poétique. (Molino, 2003, p. 304)

Le propos de cet article montre la représentation de la mer dans le récit de voyage de Pedro Montengón (1745-1824) à travers son roman didactique Eusebio (1786), grâce à l'espace cybernétique qui contient des données. Le personnage principal de ce roman est un jeune homme (Eusebio), rescapé d'un naufrage au large des côtes de Maryland en Pennsylvanie où commence son éducation dans une famille adoptive. II perd dans le naufrage ses parents, sa sœur et tous les biens embarqués dans le bateau en direction de l'Amérique, le nouveau continent où ses parents comptaient s'installer afin d'augmenter leur fortune. Une ambition freinée par le vent furieux, la violente tempête, les vagues démoniaques, le naufrage et la mort de tous les passagers excepté le jeune noble Eusebio secouru par un autre rescapé Gil Altano accroché à un mât. Nous avons une description de la mer hostile et dangereuse par opposition au littoral où les aperçoivent les valets d'un quaker, riche homme d'affaires (Henrique Myden).

Ainsi pourrons-nous examiner les détails de la mise en scène entre les tribulations des naufragés et le regard des spectateurs qui finissent par les sauver. Ce procédé de mise en scène entre le naufragé et le spectateur nous permettra d'établir une certaine correspondance de la représentation de la mer et du littoral entre Quevedo (1580-1645) et Pedro Montengón (1745-1824). Je dois en effet préciser qu'en lisant l'article de Carmen Peraita (Peraita, 2002), je me suis rendu compte qu'ils ont plus ou moins les mêmes idées qui expliquent la perte de l'homme victime des vices (l'ambition, le gain facile et du manque de retenue) qui le poussent à prendre le large par opposition aux vertus (la modération, la modestie et l'humilité) de son sauveteur (le spectateur) qui a une vie tranquille et paisible sur le littoral. De plus, c'est une philosophie qui est véhiculée par un moyen littéraire (le naufrage) à travers une technique narrative, mettant en regard la description de la mer et celle du littoral. Ainsi ai-je eu l'idée de comparer ces deux auteurs qui ont traité de la même façon les questions de leur temps, même s'ils ne sont pas de la même époque. Nous verrons que pour ces deux écrivains les causes 
de la perdition de l'être humain sont les mêmes (l'ambition, la démesure et la cupidité) et mènent à la navigation (Peraita, 2002, p. 185) souvent fatale. Il s'agira de voir le sens philosophique du procédé de perception du naufrage par le spectateur depuis la terre ferme (Peraita, 2002, p. 187). Dans une dimension plus existentielle, le point de départ est qu'on est déjà embarqué et qu'il n'y a pas de possibilité de s'abstenir : l'issue pour I'homme n'est que le sauvetage ou la noyade, d'après Blumenberg (cité par Peraita, 2002, p. 187). Bref les bateaux sont le symbole de la destinée.

En revanche, la topographie fonctionnelle (la fonction narrative de l'espace) nous permettra de mieux saisir le sens de ces différents espaces dans le roman de Pedro Montengón, que ce soit l'espace maritime, le littoral, les côtes, la grange de ses parents adoptifs en face de la mer. Cette méthode d'analyse, basée sur l'analyse de la représentation mimétique de l'espace et sur la fonction ou la dimension symbolique (ou idéologique) de l'espace en corrélation avec les actions des personnages, nous ramène à la sémiologie de l'espace. L'espace, en tant que creuset du sens dans notre démarche, permettra d'établir les similitudes entre Montengón (1745-1824) et Quevedo (1580-1645), du point de vue de la perspective narrative et de la dimension idéologique de l'espace. De fait, l'écrivain d'Alicante se sert du voyage au Nouveau Monde pour mettre, par la même occasion, en exergue ses idées romantiques, grâce à la personnification de la mer, du vent furieux, des nuages, de la nuit, de la tempête, du naufrage, de la mort, en réactualisant les idées classiques en tant qu'écrivain préromantique. Le bateau sert la narration car le naufrage provoque des « ruptures » de destinée. Il est aussi un lieu de rencontre. Le roman de Montengón l'utilise à la fois de façon ancienne (en référence au roman byzantin, c'est-à-dire la succession des évènements à travers la navigation, le naufrage permettant l'ouverture de perspectives et les rencontres) mais aussi moderne (le bateau favorise les introspections et une mise à l'épreuve morale).

En définitive, notre réflexion pourra s'articuler autour de deux principaux axes. Dans un premier temps, nous aborderons la place de Pedro Montengón dans la littérature de voyage (1.). Nous étudierons successivement dans cette première étape : I'influence de Quevedo et le rôle innovateur de Montengón dans le récit de voyage (1.1.), la mise en scène des naufragés et des sauveteurs (1.2.), ses idées préromantiques à travers la description de la mer et du littoral (1.3.), l'opposition entre le littoral et la mer (1.4), avant d'examiner dans un second temps le rôle des bateaux en tant que symbole de la destinée (2.) en s'appuyant sur le rôle narratif du naufrage (2.1.), grâce au périple (2.2.) et aux romans interpolés (2.3.). 


\section{Pedro Montengón dans la littérature de voyage}

La fonction primaire de la description est de poser l'orientation spatiale des personnages et le cadre dans lequel se situent leurs actions : elle nous fait pénétrer dans le monde du récit qu'elle construit peu à peu sous nos yeux. C'est la raison pour laquelle le récit commence souvent par l'indication, aussi succincte soit-elle, du temps et du lieu de l'action. Un cadre spatial est même plus nécessaire, plus immédiatement présent qu'une indication temporelle : les récits traditionnels se situent dans un temps éloigné mais dans un lieu précis. (Molino, 2003, p. 300)

Le XVIII siècle, ou plutôt le siècle des Lumières, est un siècle qui vient bouleverser l'ordre des choses à travers toute l'Europe, par la quête de la Liberté et du Bonheur en dehors de la religion catholique, mise à part l'Espagne qui s'est repliée sur elle-même pour se préserver de l'éclosion des nouvelles idées par le biais du Saint Office ou Tribunal de la foi. C'est pourquoi Pedro Montengón (1745-1824) s'est confronté à des problèmes d'ordre religieux à la suite de la publication de son roman intitulé Eusebio (1786). En fait, il s'agit d'un roman qu'il a écrit après son exil en Italie. Rappelons qu'il s'est exilé en 1767 à la suite du décret d'expulsion des jésuites ordonné par Charles III (Charles de Bourbon, roi d'Espagne, de Naples et de Sicile).

Pedro Montengón fait partie des meilleurs écrivains du XVIII' siècle espagnol en jouant un rôle capital dans la littérature de voyage dont nous pouvons citer, entre autres auteurs incontournables, Cadalso avec Las cartas marruecas et Mélendez Valdés avec Las cartas turcas. Selon Francisco Lafarga (Lafarga, 1994, p.10), les voyages n'étaient pas prisés par les Espagnols, à cette époque. Cela constitue un paradoxe, au regard d'une Europe marquée par la tendance aux voyages pour connaître les pays voisins, voire en dehors de leur continent, à savoir l'Amérique. II y a lieu de rappeler que la littérature de voyage était limitée en Espagne. L'Amérique, I'Italie, l'Angleterre, et surtout la France sont les principales destinations (Lafarga, 1994, p. 5).

Cependant, il faut signaler que les vraies raisons de ces voyages n'étaient pas l'esprit de curiosité (Lafarga, 1994, p. 18) mais celui de la connaissance et de la vulgarisation de ces mêmes connaissances visant à éduquer leurs compatriotes repliés sur leur terre et réfractaires aux nouvelles idées qui circulaient en Europe. Ainsi Montengón, Cadalso et Meléndez Valdez ont largement contribué au changement de mentalité et de goût 
qui était I'unique préoccupation des écrivains de la fin du XVIII siècle. Autrement dit, ils ont participé à l'accélération du passage du XVIII ${ }^{\mathrm{e}}$ au XIXe siècle.

\subsection{Récit de voyage : I'influence de Quevedo et le rôle innovateur de Pedro Montengón}

La mer, la navigation, la tempête et le naufrage sont des clichés de prédilection dans la poésie morale de Quevedo (Peraita, 2002, p. 181). Ce sont des métaphores employées dans différents contextes à plusieurs caractéristiques, avec des significations et des valeurs diverses. Sa poésie exprime les tribulations de l'être humain et la fugacité du temps (Peraita, 2002, p. 182). Naviguer signifie traditionnellement enfreindre les lois de la nature, violenter la terra inviolata, en s'exposant à des risques inutiles, selon toujours Carmen Peraita. La condamnation de la navigation est un topique de la tradition du monde gréco-latin (Peraita, 2002, p.183), mais Quevedo y associe la tradition chrétienne dont l'iconographie chrétienne diabolise la mer : celle-ci devient dès lors quelque chose d'imprévisible, où il est difficile de trouver un chemin. Elle est un espace où se manifestent le mal et le démon (Peraita, 2002, p. 183-184).

Dans le roman de Pedro Montengón (Eusebio), nous notons la présence de Dieu à travers des expressions comme la Providence, envers qui Eusebio exprime sa gratitude de l'avoir sauvé d'un naufrage redoutable et funeste (Montengón, 1998, p. 311). Une manière pour Pedro Montengón d'affirmer sa croyance en la foi chrétienne, rejetant ainsi toutes les accusations d'impiété qui lui ont été faites pour avoir mis en avant la vertu naturelle (doctrine des quakers basée sur la raison qui mène au bonheur à l'image de la nature pure vierge, par opposition au vice) comme le socle de la religion. II y a donc une coïncidence entre les idées de Quevedo et de Montengón si l'on s'en tient à la représentation de la navigation et du naufrage, dans la mesure où Eusebio évoque la Divinité en ces termes: Main Omnipotente, Ciel, Providence, le Seigneur Juste et Clément, I'Éternel Artifice et Créateur Suprême (Pérez-Rioja, 1988, p. 14-15). En outre, la navigation dans la poésie de Quevedo est un signe d'insatisfaction de l'homme par rapport à son existence (Peraita, 2002, p. 185). L'ambition et la démesure provoquent la perte de I'homme qui est à la recherche effrénée de richesses, quitte à braver les dangers de la traversée de la mer (Peraita, 2002, p. 185). Dans Eusebio de Montengón, nous avons cette même représentation de l'ambition et de la cupidité de l'homme, car les parents du jeune Eusebio sont des nobles qui souhaitaient augmenter leur fortune en Amérique, avant le naufrage fatidique. 
En définitive, il existe également une autre coïncidence entre Quevedo et Montengón en ce qui concerne la mise en scène du naufrage, et surtout le spectateur imperturbable qui observe l'affliction d'autrui à partir du littoral (Peraita, 2002, p. 186). Il y a lieu de préciser qu'il s'agit d'un procédé classique : la représentation du danger de la mer est en contradiction avec la paix de la maison, la sécurité du port, (et plus particulièrement chez Quevedo) la maîtrise des passions. Cependant Hans Blumenberg, (cité par Peraita, 2002, p. 187) a fait des recherches sur les implications philosophiques de la réception de la métaphore du spectateur de naufrage en rapport avec la distance de la terre ferme. Hans Blumenberg (1995, p. 14) examine dans ses travaux le paradoxe de la métaphore que les êtres humains qui vivent sur la terre aiment dans leur imagination représenter leur condition générale dans le monde à travers une traversée de la mer. La métaphore de la vie comme navigation renferme de nombreuses conditions et de possibilités, donnant une sorte d'ébauche ou de schéma d'un tout : le «port » et ses alentours « étranges », les « pilotes», les « gouvernails», les « ancres», les « golfes», la «mer », les « voiles», les « tempêtes», les « vents » et le « calme ». C'est un modèle qui doit être soumis à de multiples actualisations, à des formes variées dans lesquelles les êtres humains imaginent leur relation avec le monde. Cette actualisation fréquente est celle du spectateur qui observe la calamité de ceux qui font naufrage.

\subsection{La mise en scène des naufragés et des sauveteurs}

Il y a lieu de s'interroger sur la technique narrative afin de mieux comprendre la mise en scène des naufragés et des sauveteurs. De prime abord, nous avons une description éloignée d'un objet flottant, avant d'être beaucoup plus précis aux yeux des domestiques d'un riche homme d'affaires (Henrique Myden). Les sauveteurs sortent de la ferme qui se trouve à côté de la plage. Leur objectif est de faire le point sur les dégâts de la violente tempête de la veille. Mais pour «motiver » et rendre naturelle l'entrée dans la fiction, le narrateur hétérodiégétique se sert d'une série de topoï utilisés par les romanciers (Jouve, 1997, p. 21-22). C'est le topos de «l'inconnu » qui permet au narrateur de cacher sa présence en faisant semblant de ne rien connaître de ses personnages (les deux rescapés dans Eusebio). C'est ainsi que le lecteur devient un personnage découvrant grâce à ce topos. Pennsylvanie et les autres toponymes du roman de Pedro Montengón sont présentés de manière naturelle dans l'univers fictif, dès l'incipit, sans oublier enfin le topos « de dévoilement » consistant à dévoiler progressivement et « naturellement » l'espace de l'histoire, notamment la description de l'aube, du lever du jour au tout début du roman (Montengón, 1998, p. 87). 
C'est ainsi que le narrateur poursuit son récit en évoquant la ville de Salem, située à côté du fleuve Delaware, lieu de résidence du maître Hardyl, c'est-à-dire de celui qui va servir d'interprète entre Henrique Myden et ses hôtes (Montengón, 1998, p. 90), rescapés d'un naufrage survenu la veille au large des côtes.

\subsection{La description de la mer et du littoral : les idées préromantiques de Pedro Montengón}

La description des impacts de la tempête sur la mer et le littoral permet à l'auteur de véhiculer des idées romantiques en tant qu'écrivain de la fin du XVIII et du début du XIX'. Sa particularité est qu'il se situe entre deux siècles. C'est pourquoi Rogelio Blanco défend la thèse selon laquelle Montengón a un « caractère innovateur » et une avance sur les « contenus préromantiques » et triomphants du XIX siècle, tout en gardant la «force » et la « capacité de la raison » (Banco, 2001, p. 349). Ainsi nous donnet-il beaucoup d'exemples de composantes romantiques facilement repérables à travers l'œuvre de Montengón : « le désir ardent de liberté », « la clameur contre l'injustice », «l'amour sensuel », « le sentimentalisme », «l'éloge du héros », « la présence des larmes », «la tristesse et la mélancolie », « les paysages naturels abrupts », « la chute des torrents dans les rocs escarpés », « le vent furieux », " la mer courroucée », « la révolte », « la solitude », " le refuge à la campagne », « la nostalgie d'un passé heureux ", « le désir ardent de la liberté », « la désillusion amoureuse », « la nuit », « la mort », « les tombes », et enfin la « passion effrénée».

Enfin, pour mieux comprendre le naufrage dans le roman de Pedro Montengón, nous examinerons tour à tour la mise en regard de l'espace du littoral avec celui de la mer, la symbolique des bateaux dans le récit de voyage, la fonction narrative du naufrage (que ce soit dans les différents voyages du protagoniste, ou dans les romans intercalés).

\subsection{L'opposition entre littoral et mer : paix, fertilité contre orgueil, ambition et perdition}

La grange d'Henrique Myden se situe dans une campagne paisible, tranquille, fertile et paradisiaque contrairement à la mer dangereuse et démoniaque où se dissipent facilement la volonté et l'ambition des Européens qui prennent le risque de la braver en la traversant afin d'augmenter leur fortune grâce aux ressources naturelles de I'Amérique. La mer toujours périlleuse nous révèle les conditions difficiles dans lesquelles 
voyagent les soldats de l'aventure qui sont toujours victimes de naufrages mortels dans le roman de Pedro Montengón, à l'exception des protagonistes (l'élève Eusebio et son maître artisan Hardyl, sans oublier leurs serviteurs) formés dans la vertu naturelle dont le socle est la modération, la modestie et l'humilité.

L'espace caractérise les personnages au vu des difficultés rencontrées par le maître Hardyl lorsqu'il apprend à son élève Eusebio le métier de vannier pour faire disparaître son orgueil provoqué par la luxueuse villa de son père adoptif à Philadelphie.

Malgré le danger des traversées, l'Émile espagnol s'en sort toujours indemne. C'est une manière aussi pour le narrateur de nous suggérer en filigrane les dangers de l'orgueil, de l'ambition qui sont à l'origine de l'Âge de fer d'une Europe sclérosée à cause des vices de la noblesse oisive, entichée de leur noblesse par opposition à une noblesse de mérite basée sur le travail et la vertu naturelle symbolisée dans Eusebio par l'Amérique, et surtout par la campagne à travers la grange ou le lieu de sauvetage du jeune noble Eusebio et de son valet Gil Altano. Toute proportion gardée, la ville (où prédominent l'orgueil et l'ambition) incarne également le danger qui peut être mis en regard avec la mer à l'opposé du littoral édénique, idéalisé.

Aussi avons-nous remarqué tout au long du périple de nos protagonistes que les villes sont d'une manière générale les lieux où se dégrade leur situation alors que les campagnes constituent un havre de paix. En clair, dès l'incipit du roman, le narrateur nous décrit une campagne où prédominent l'espoir, la tranquillité et une terre très fertile. Cette campagne est source de richesses au vu de la fortune d'Henrique Myden, homme d'affaires à Philadelphie. Philadelphie est évidemment la ville où commencent à apparaître les difficultés concernant l'éducation d'Eusebio, à cause de l'orgueil qui ne fait que provoquer l'ambition contrairement à la campagne où Eusebio et Gil Altano ont été sauvés après le naufrage, c'est-à-dire les côtes de Caroline en Floride.

\section{Les bateaux : symbole de la destinée}

Les bateaux nous suggèrent de manière générale les conditions difficiles et périlleuses dans lesquelles les différents voyageurs du roman de Pedro Montengón (1745-1824) ont tous traversé une mer (tantôt calme, tantôt démontée), sans oublier les aléas climatiques qui pouvaient faire basculer le destin des candidats à l'aventure. Nous avons comme exemple le voyage de la famille d'Eusebio qui s'est mal terminé. Autrement 
dit, c'est un voyage qui a engendré une tragédie : toute une famille est presque décimée au large des côtes de Floride. Il va sans dire que les voyages sont risqués, en raison de la longue distance qui sépare les deux continents, c'est-à-dire l'Europe et l'Amérique. Comme nous avons déjà beaucoup insisté sur le naufrage survenu dès le début du roman, nous pouvons évoquer les autres voyages à bord de bateaux. Dans le roman d'aventure, il figure la destinée. Plus généralement, le voyage en bateau est un artifice narratif qui ouvre des possibilités.

\subsection{Le naufrage : rôle narratif, rupture et ouverture de possibilités}

\subsubsection{Le périple et les souvenirs du naufrage}

Lors du deuxième voyage, c'est-à-dire le périple avec son maître Hardyl, Eusebio, une fois dans le bateau, rumine les mauvais souvenirs du naufrage. Toutefois, devant les contingences de la vie, le narrateur omniscient nous décrit l'état d'esprit d'un Eusebio effaré, mais vite rassuré par la toute-puissance de la Providence qui l'a déjà sauvé d'un naufrage qualifié de redoutable et de funeste. Ce début de voyage met à nu la foi inébranlable en Dieu d'un jeune homme élevé dans un milieu où prédomine la religion naturelle. De fait, la dangerosité de la mer met en relief le procédé utilisé par Pedro Montengón pour mettre en avant la religion chrétienne sous-tendue par sa croyance en la vertu naturelle. L'évocation de la reconnaissance de son salut envers la « providence » est un moyen qui lui a permis de réfuter les préjugés d'impiété dont il a été victime :

A bord du bateau, Eusebio pouvait à peine apercevoir les montagnes les plus élevées de l'Amérique depuis le large et le bateau sillonnait la mer à travers un vent frais ; mais il avait encore à l'esprit l'endroit où il pensait laisser sa bien-aimée Leocadia. Elle ne cessait de troubler ses pensées ; la crainte qu'il ressentait lorsqu'il se voyait livré aux aléas climatiques était aussi bien liée au risque encouru qu'à la perte de sa bien-aimée. Ce qui lui faisait penser, alors, à un naufrage plus redoutable et plus funeste que celui dont la providence l'a délivré. (Montengón, 1998, p. 311)

Une fois tranquillisé, Eusebio craint le pire avec le mauvais temps qui se déclenche : les nuages s'amoncellent et le soleil devient presque invisible. De plus, le vent siffle et les vagues de la mer redoublent d'intensité avec une violence inouïe face à une embarcation fragile : 
Des amas de nuages noirs s'accumulaient dans le désordre de l'atmosphère. Pâle et craintif, le soleil avait l'air d'être couvert par un épais voile pour ne pas voir les malheurs qui se profilaient à I'horizon. Le vent augmentait ; les amarres les plus puissantes grinçaient avec beaucoup de bruits. De plus en plus démontée, la mer grognait et frappait avec la plus grande force le bateau fragile en le couvrant de ses vagues. Eusebio ne peut résister à un spectacle si horrible que lui offre la violente tempête : il va à la poupe assener le capitaine de questions. (Montengón, 1998, p. 312)

Cette étape marque un tournant dans le voyage en mer, puisqu'il s'agit d'une épreuve inversée, c'est-à-dire le naufrage en Amérique et l'arrivée à bon port en Angleterre. Pour le lecteur, il y a aussi une incertitude narrative et une création de suspense.

\subsubsection{Le naufrage dans les romans intercalés}

Dans ces romans intercalés, nous avons le même scénario que l'histoire des parents d'Eusebio : la volonté de voyager en Amérique pour s'enrichir davantage en augmentant leurs richesses. C'est une série de punitions de l'ambition de ces personnages secondaires. Une manière de varier les histoires d'infortune causées par la cupidité, permettant à Pedro Montengón de joindre l'utile à l'agréable. Ces naufrages à répétition que nous verrons successivement sont les conséquences d'un vice qui sert de contrepoint à la vertu (la modestie, I’humilité et la retenue).

Le jeune Bridge a connu à son tour le naufrage d'un navire qui est en partance pour le Québec lors de sa fuite de l'Angleterre, c'est-à-dire à partir de Plymouth pour échapper à la justice après avoir assassiné le fils du lord Ut. C'est un naufrage qui a fait plus de peur que de mal cette fois-ci parce qu'il n'y a pas eu de mort, mis à part la perte de la fortune de ce jeune Bridge et de toute la provision du bateau. Ce dernier incrimine le pilote du navire pour avoir fait preuve de négligence totale au moment où il s'apprêtait à accoster le port du Québec :

Je voyageais avec un immense capital pour espérer avoir très facilement un meilleur sort ; mais ce dernier se moque de la sécurité qui fonde l'espoir des hommes, bien que le bateau se soit déplacé sans problème, il n'a pas voulu que je profite de mon trésor en l'ensevelissant dans la mer lorsque nous nous dirigions vers le port et que le navire heurta un récif par 
négligence du pilote. [...] Les passagers se sont sauvés, excepté le navire et mon argent qui sont restés prisonniers des vagues. C'est ainsi que je suis arrivé au Québec, pauvre et ruiné. (Montengón, 1998, p. 131-132)

Dans une autre histoire intercalée, le voyage en mer du lord Towsend s'est bien passé. Autrement dit, le temps était relativement calme. Il faisait donc beau. Mais, le seul souci du lord Towsend, c'est le sort de ses deux jeunes filles à bord de ce petit bateau conduit par Tautel qui ne cesse de le rassurer malgré le long voyage :

C'est ainsi qu'avons passé toute la nuit sans dormir et mon esprit se faufilait à travers mille pensées funestes, beaucoup plus rapide que le skiff sur les paisibles vagues; le vaillant Tautel, les rares fois qu'il abandonnait les rames sur le tolet, a beau essayer de nous consoler en nous faisant croire à la proximité de notre refuge sûr, où nous venions d'arriver. (Montengón, 1998, p. 680)

Dans une troisième histoire intercalée, Jacobo Camder était le capitaine de la caraque, c'est-à-dire du navire (« una carraca »), (Montengón, 1998, p. 90), selon les dires de Gil Altano qui ne cessait de le remercier pour lui avoir sauvé la vie et épargné les sévices que ses hommes ont voulu lui infliger. En effet, Gil Altano et ses collègues sont secourus par ce capitaine après le naufrage du bateau à bord duquel il voyageait comme matelot à destination de Buenos Aires. Par conséquent, c'est un naufrage lié au mauvais temps:

Comment; vous ne vous souvenez pas, mon seigneur, de ce galion-là qui allait à Buenos Aires, il y a juste quatre ans, et que vous nous aviez pris en chasse et rattrapés au moment où il coulait à cause des grandes vagues? [...] C'est à bord de ce galion, donc, continua Gil Altano, que j'étais marin et que j'ai alors bénéficié de votre généreuse humanité, mon seigneur, en nous faisant monter à bord de votre rafiot, où vos marins voulaient nous ligoter en nous traitant alors comme des prisonniers de guerre; et mon seigneur, vous ne l'avez pas accepté. (Montengón, 1998, p. 91)

De manière générale, le bateau sert la narration car le naufrage provoque des « ruptures » de destinée. C'est un moyen narratif qui met en exergue les contingences de la vie et les risques du voyage en mer. Il est aussi un lieu de rencontres. Le roman 
de Montengón l'utilise à la fois de façon ancienne (en référence au roman byzantin) mais aussi moderne (le bateau favorise les introspections et une mise à l'épreuve morale).

Ce sont donc des indices de l'espace qui servent à renforcer la vraisemblance du récit, tout au long du roman. Ce qui est remarquable, c'est que le soleil, le vent, la mer sont personnifiés dans la perspective de mieux faire ressortir le caractère redoutable de la tempête subie par Eusebio, Hardyl et leurs compagnons de voyage.

\section{Conclusion}

Les bateaux et le naufrage expriment la condition humaine à travers les contingences de la vie, la caducité des biens matériels, les dangers de la traversée maritime. Le naufrage est un artifice littéraire qui a permis au narrateur de nous présenter un homme neuf (Eusebio, un enfant de six ans) destiné à l'éducation de la vertu naturelle, juste après la perte de ses parents nobles qui ont voulu se rendre au Nouveau Monde pour augmenter leur fortune. Autrement dit, la passion effrénée pour les richesses et l'ambition sont des vices qui peuvent facilement perdre toute personne qui n'est pas éduquée dans la vertu naturelle des quakers qui s'étaient exilés en Amérique (Pennsylvanie) afin de fuir l'intolérance religieuse de l'Europe.

L'Amérique incarne dans le roman de Montengón l'Âge d'Or et l'Europe l'Âge de fer. La grange d'Enrique Myden qui se situe au niveau des côtes de Maryland est un espace édénique propice à l'éducation d'Eusebio contrairement à la ville où règnent beaucoup d'obstacles à cause de l'orgueil et de l'ambition.

Somme toute, ce ne serait pas absurde de lire dans le roman de Pedro Montengón ses conditions de vie difficiles lors de son exil en Italie, juste après l'expulsion des Jésuites, voire sa traversée de la mer en direction de l'Italie, tout comme Quevedo, (Peraita, 2002, p. 195), si l'on tient compte de ses propres naufrages existentiels pendant son emprisonnement à San Marcos où il a été assailli par les tempêtes et les menaces de noyade. C'est en ce sens qu'on peut penser que ces deux écrivains, éloignés de leur pays, méditent et livrent une lecture de leur propre passé. 
Évaluation : Évaluation anonyme par des pairs extérieurs.

Conflit d'intérêts : L'auteur n'a aucun conflit d'intérêts à déclarer.

Subvention : L'auteur n'a reçu aucun soutien financier pour ce travail.

Peer-review: Externally peer-reviewed.

Conflict of Interest: The author has no conflict of interest to declare.

Grant Support: The author declared that this study has received no financial support.

\section{Bibliographie}

Blanco, R. (2001). Pedro Montengón y Paret (1745-1824): un ilustrado entre la utopía y la realidad, p. 349, Universidad Politécnica de Valencia.

Jouve, V. (1997). La Poétique du Roman. Paris : Sedes.

Lafarga, F. (1994). Territorios de lo exótico en las letras españolas del siglo XVIII, Anales de Literatura Española [Publicaciones periódicas] Numéro 10, p. 10, Universidad Pompeu Fabra. Récupéré du site :http://www. cervantesvirtual.com/FichaObra.html?Ref = 20686

Montengón, P. (1998). Eusebio. Edición de Fernando García Lara. CÁTEDRA, Letras Hispánicas.

Molino, J. (2003). Homo Fabulator, Espace et Description. Paris : Actes Sud. p. 283-313.

Peraita, C. (2002), Espectador del naufragio. Muestra en oportuna alegoría la seguridad del estado pobre y el riesgo del poderoso, La Perinola, 6, p. 181-195, Villanova University, Pennsylvania. Recuperé sur le site: http: // biblioteca.org.ar/libros/200458.pdf

Pérez-Rioja, J. A. (1988) Un Best-seller del siglo XVIII: la novela «Eusebio», de Montengón, Artes Gráf. Municipales, p. 14-15, Madrid. 
